\section{Uso de álcool e drogas e sua influência sobre as práticas sexuais de adolescentes de Minas Gerais, Brasil}

\author{
Alcohol and illicit drug use and its influence \\ on the sexual behavior of teenagers from \\ Minas Gerais State, Brazil
}

\author{
Neilane Bertoni ${ }^{1}$ \\ Francisco I. Bastos 1 \\ Maeve Brito de Mello 1,2 \\ Maria Yolanda Makuch 2 \\ Maria Helena de Sousa 2 \\ Maria José Osis 2 \\ Anibal Faúndes 2
}

\begin{abstract}
This article summarizes the findings of a survey including 5,981 students from public schools in Minas Gerais State, Brazil. The analysis assessed the influence of drug use on sexual practices. Among the boys engaged in relationships with casual partners who stated having used illicit drugs, 55.7\% reported consistent condom use, as compared to $65.4 \%$ among those not reporting such habits. Among boys engaged in relationships with stable partners who reported illicit drug use, consistent condom use was reported by $42.7 \%$, versus $64.1 \%$ among those not reporting such habits. In the subgroup of boys engaged in stable relationships who did not report illicit drug use, consistent condom use was less frequent among those that used alcohol/cigarettes, compared to those who did not drink or smoke (60.7\% vs. 71.1\%). Girls were less likely than boys to use condoms consistently, regardless of the nature of their relationships, without a noticeable influence of drug use. Policies to prevent drug abuse, sexually transmitted diseases, and unplanned pregnancy should be fully integrated.
\end{abstract}

Alcohol Drinking; Street Drugs; Sexual Behavior; Adolescent

\section{Introdução}

O consumo de substâncias que alteram o psiquismo e a percepção não é um fenômeno exclusivo da modernidade, mas sim algo que perpassa toda a história da humanidade 1 . Antes restrito a determinados segmentos populacionais e circunstâncias, disseminou-se por uma ampla gama de estratos sociais e contextos, constituindo, hoje, uma questão de saúde pública ${ }^{2}$.

Estudos realizados junto a estudantes pelo Centro Brasileiro de Informações sobre Drogas Psicotrópicas (CEBRID) evidenciam que a adolescência é a fase da vida em que, em virtude da maior exposição e vulnerabilidade aos efeitos nocivos resultantes do uso de substâncias psicoativas (sejam elas ilícitas, como a maconha e a cocaína, ou lícitas, como o álcool), a experimentação destas torna-se um fenômeno mais freqüente e, eventualmente, definem-se padrões de consumo repetitivo, que podem estar associados a diferentes riscos e danos 3,4 .

Além disso, aspectos relacionados à sexualidade ganham igualmente relevância entre os adolescentes, no contexto da saúde pública, ressaltando-se, dentre eles, a gravidez precoce $5,6,7,8,9$ e o risco de aquisição de infecções sexualmente transmissíveis (IST) 10,11. O uso de preservativos, apesar de se mostrar mais freqüente entre os jovens brasileiros em anos recentes 12 , não se dá de forma consistente em todas as relações 13 , aumentando, com isso, as chances de gravidez 
indesejada e da aquisição de IST, inclusive a infecção pelo HIV 10,11.

Reconhecendo a complexidade das inter-relações entre adolescência, consumo de drogas e sexualidade, este estudo analisa, de forma exploratória, comportamentos de uma amostra de 5.981 jovens (10-19 anos), matriculados em escolas públicas de 12 municípios de Minas Gerais, Brasil, com relação ao uso de drogas, na sua inter-relação com o uso de preservativos nas suas práticas sexuais.

\section{Métodos}

O presente estudo apresenta achados referentes à linha de base da pesquisa de avaliação do Programa de Educação Afetivo-Sexual, Um Novo Olhar (PEAS), desenvolvido pelas Secretarias Estaduais de Saúde e Educação de Minas Gerais junto a estudantes do 7o. ao 9o. ano do Ensino Fundamental e da 1a a 2a séries do Ensino Médio de escolas públicas do Estado de Minas Gerais. Originalmente, os 41 municípios-sede das secretarias estaduais foram selecionados para participar do programa; destes, 12 (Caratinga, Divinópolis, Governador Valadares, Januária, Manhuaçu, Montes Claros, Muriaé, Paracatu, Patos de Minas, Teófilo Otoni, Uberaba e Uberlândia) foram escolhidos para o processo de avaliação com base na proporção de sua população urbana - maiores e menores valores dessas proporções pautados em dados do Censo Demográfico de 2000 (Instituto Brasileiro de Geografia Estatística; http:/ / www.ibge.gov. br), para cada micro-região do Estado de Minas Gerais - e maior número de escolas públicas.

O estudo utilizou amostragem por conglomerados. Em cada cidade, foram escolhidas quatro escolas (unidades primárias de amostragem), mediante aceitação das respectivas Direções em participar do programa (grupo 1), que foram pareadas a quatro outras escolas com perfil sócio-demográfico semelhante, de acordo com a Secretaria Estadual de Educação, e que fossem próximas geograficamente das primeiras, para que servissem como grupo de comparação (grupo 2).

Em seguida, foram sorteadas 19 salas de aula (unidades secundárias de amostragem), para cada um dos grupos acima, do 7o ano do Ensino Fundamental à 2a série do Ensino Médio, por amostragem sistemática simples, utilizando-se duas listas completas elaboradas para esse fim, contendo todas as turmas dessas séries nas quatro escolas-programa, bem como nas escolascomparação. Dessa forma, considerando-se as 12 cidades - cada uma delas com dois grupos de escolas (as que participariam do PEAS e as que não participariam), 19 salas de aula sorteadas, com aproximadamente 35 alunos cada uma, e a estimativa de uma taxa de resposta geral de $40 \%$ - a previsão era de se atingir aproximadamente 6.400 alunos na amostra do levantamento de base.

A 3a série do do Ensino Médio não foi incluída, uma vez que um dos objetivos centrais do projeto mais abrangente (PEAS), lançado em 1999, era o de proceder a avaliações longitudinais das escolas a partir do lançamento do referido programa. Portanto, a avaliação poderia ser feita apenas para alunos a partir do 70 ano, ou seja, os alunos de 6 o ano quando do lançamento do PEAS, em 199914.

Todos os alunos matriculados nessas classes foram convidados a responder a um questionário de múltipla escolha, auto-aplicado, prétestado e validado junto à população-alvo 14 . $\mathrm{O}$ instrumento continha 43 perguntas, de natureza sócio-demográfica e relativas ao conhecimento, atitudes e práticas de risco para o HIV e gravidez precoce. Embora nenhum questionário contivesse a identificação pessoal do entrevistado, todos os alunos de cada turma responderam ao questionário, com o intuito de não definir subgrupos que poderiam ser identificados pelos alunos como "sexualmente ativos" e "sexualmente inativos". Como o estudo estava voltado apenas para os alunos que tiveram experiência sexual previamente à aplicação do instrumento, apenas estes foram incluídos na análise. Os dados da linha de base foram coletados entre 25 de setembro e 1 o de dezembro de 2000, compreendendo uma amostra de 6.858 alunos. A participação dos estudantes foi voluntária, mediante autorização por escrito dos responsáveis e assinatura de um termo de consentimento livre e esclarecido. O protocolo do estudo foi aprovado pelo Comitê de Ética da Faculdade de Ciências Médicas da Universidade Estadual de Campinas.

Após aproximadamente 18 meses, esse mesmo questionário foi aplicado em outra amostra composta por 7.613 alunos das mesmas escolas como parte do desenho de avaliação do programa. Cabe observar, entretanto, que os dados referentes a esta segunda fase de avaliação não constituem objeto do presente trabalho.

Foram excluídos 877 questionários da linha de base por terem sido respondidos inadvertidamente por adolescentes pertencentes a turmas não sorteadas, por alunos que não estavam incluídos na faixa etária definida de antemão (10-19 anos), ou que não informaram sua idade. Com isso, o total de questionários válidos para a presente análise foi de 5.981 .

Na primeira etapa, a amostra estudada foi caracterizada quanto a variáveis sócio-demográfi- 
cas, aos padrões de consumo de drogas e álcool e ao uso de preservativo conforme o tipo de parceria em que os entrevistados estavam envolvidos - parceiros fixos (definidos como "pessoa(s) com quem você tem relações sexuais regularmente, por exemplo, em um namoro, casamento ou caso") ou com parceiros eventuais (definidos como "pessoa(s) com que você fica ou ficou e teve/tem relação sexual”).

Posteriormente, foi empregado o procedimento de árvores de classificação, denominado chi-squared automatic interaction detector (CHAID), uma modalidade de análise discriminante que permite particionar amostras e caracterizar subgrupos de indivíduos com comportamentos similares/dissimilares 15 , com o intuito de investigar possíveis associações entre o uso de drogas e/ou álcool e a adoção de práticas sexuais não seguras.

A presente análise considerou comovariáveisdesfecho a serem avaliadas: "uso de preservativo com parceiro casual" e "uso de preservativo com parceiro fixo" (dicotomizado em "uso consistente/não consistente"). A categoria "uso consistente do preservativo" refere-se às respostas em que os participantes relataram sempre usar preservativo nas suas relações sexuais. A categoria "uso não consistente do preservativo" diz respeito às demais respostas, ou seja, àquelas em que os entrevistados referiram usar o preservativo $n a$ maioria das vezes, de vez em quando ou nunca.

O tamanho de cada subgrupo foi limitado em virtude de, eventualmente, não haver associação estatisticamente significativa entre a variável analisada e a variável-desfecho. Foi ainda realizado o procedimento de validação cruzada dos achados, com fator 10 (correspondendo ao número de vezes em que cada iteração é repetida), a fim de obter resultados mais confiáveis 15 . As observações com valores faltantes/ignorados foram excluídas da análise.

As co-variáveis analisadas por meio do procedimento CHAID, na sua possível associação com as variáveis-desfecho, foram: sexo (feminino/masculino), faixa etária (10-14 anos/15-19 anos), uso de droga lícita (nunca usou/usou ou usa) e uso de droga ilícita (nunca usou/usou ou usa).

Por drogas lícitas entenda-se aqui álcool e/ ou cigarro; por drogas ilícitas, maconha, cocaína ou outro tipo de droga (não lícita) referida pelos entrevistados. Essas variáveis, que, a princípio, tinham como opções de resposta: "nunca usei", "só experimentei”, "uso às vezes", "uso sempre” e "usei, mas não uso mais", foram recategorizadas em "nunca usou" e "usou ou usa", por causa do pequeno número de observações constantes de cada categoria.

\section{Resultados}

A amostra estudada era composta por $40,2 \%$ de adolescentes do sexo masculino e 59,6\% de adolescentes do sexo feminino. Quando questionados a respeito de sua cor, $29,2 \%$ dos entrevistados se classificaram como brancos, enquanto $61,2 \%$ descreveram-se como morenos e $9 \%$ como negros/mulatos/outra cor. Com relação à idade, a distribuição dos entrevistados nos dois grupos etários, 10-14 anos e 15-19 anos, foi praticamente homogênea, com $48,3 \%$ e $51,7 \%$, na primeira e segunda faixas etárias, respectivamente. Apesar disso, a maior parte dos alunos, aproximadamente $73 \%$, cursava do 7o ao 9o ano do Ensino Fundamental, refletindo o fato de que muitos adolescentes estavam em séries abaixo daquela esperada para a sua idade. Cerca de $61 \%$ dos adolescentes da amostra $(\mathrm{n}=3.667)$ estudavam no período da manhã; as porcentagens de alunos da amostra nos turnos da tarde e noite foram semelhantes, a saber, $19 \%$ e $19,6 \%$, respectivamente (Tabela 1).

Com relação a todos os tipos de drogas, a proporção de adolescentes que referiram usar com freqüência (usa às vezes/usa sempre) foi mais elevada para a faixa etária de 15-19 anos. Porém, os dados apresentados na Tabela 2 chamam a atenção para as porcentagens relativamente elevadas de uso destas substâncias por parte dos adolescentes de 10-14 anos. Dentre estes últimos, $45,9 \%$ relataram já ter feito/fazer uso de álcool; $23 \%$, de cigarro; $2,4 \%$, de maconha; $3,7 \%$ de outra droga ilícita (que não a maconha).

A freqüência do uso de cada droga foi estratificada por gênero, segundo faixa etária, sendo aplicado teste $\chi^{2}$ de independência entre essas variáveis para cada uma das drogas analisadas (Tabela 2). Em relação ao consumo de álcool, não foi observada diferença significativa entre rapazes e moças na faixa etária de 10-14 anos; de modo distinto, na faixa de 15-19 anos, essas diferenças se mostraram estatisticamente significativas, com claro predomínio dos rapazes, que utilizavam mais freqüentemente o álcool (usa às vezes/usa sempre), se comparados às moças da mesma faixa etária $(\mathrm{p}=0,000)$.

O consumo do cigarro e de drogas ilícitas (que não a maconha) mostrou-se mais prevalente entre os meninos do que entre as meninas, contudo essa diferença não se mostrou estatisticamente significativa ( $p>0,05)$. Já com relação ao uso da maconha, as adolescentes do sexo feminino referiram menor proporção de uso dessa substância, se comparadas aos rapazes da mesma faixa etária $(\mathrm{p}<0,01)$.

Aproximadamente dois terços dos adolescentes $(3.875$ ou $65 \%$ - sendo $25 \%$ destes do se- 
Características sócio-demográficas e contextuais de adolescentes de 12 municípios de Minas Gerais, Brasil, 2000.

\begin{tabular}{|c|c|c|}
\hline Variável & $\mathbf{n}$ & $\%$ \\
\hline \multicolumn{3}{|l|}{ Sexo } \\
\hline Masculino & 2.405 & 40,2 \\
\hline Feminino & 3.563 & 59,6 \\
\hline Não-resposta (missing) & 13 & 0,2 \\
\hline \multicolumn{3}{|l|}{ Cor (auto-referida) } \\
\hline Branca & 1.748 & 29,2 \\
\hline Morena & 3.661 & 61,2 \\
\hline Negra/Mulata/Outra & 536 & 9,0 \\
\hline Não-resposta (missing) & 36 & 0,6 \\
\hline \multicolumn{3}{|l|}{ Faixa etária (anos) } \\
\hline $10-14$ & 2.888 & 48,3 \\
\hline $15-19$ & 3.093 & 51,7 \\
\hline \multicolumn{3}{|l|}{ Escolaridade (em curso) } \\
\hline 7으 ao 9ㅇa ano do Ensino Fundamental & 4.363 & 73,0 \\
\hline 1ạ a 2a série do Ensino Médio & 1.617 & 27,0 \\
\hline Não-resposta (missing) & 1 & 0,0 \\
\hline \multicolumn{3}{|l|}{ Período em que estuda } \\
\hline Manhã & 3.667 & 61,3 \\
\hline Tarde & 1.134 & 19,0 \\
\hline Noite & 1.175 & 19,7 \\
\hline Não-resposta (missing) & 5 & 0,1 \\
\hline
\end{tabular}

xo masculino e $75 \%$ do sexo feminino) referiram nunca ter tido relações sexuais. Dos que já haviam se iniciado sexualmente, a média de vida sexual ativa foi de 2,74 anos para os meninos e de 1,34 ano para as meninas (desvio-padrão $=2,17 \mathrm{e}$ 1,47 , respectivamente; dados não apresentados em tabela).

Com relação ao uso consistente de preservativo, as proporções variaram de acordo com a natureza da parceria e sexo. Dos 1.307 adolescentes que disseram ter tido relações sexuais com parceiro casual, o uso consistente de preservativo foi relatado por $63,7 \%$ dos homens (575/903) e por $49,8 \%$ das mulheres $(201 / 404)$. Em se tratando de parceria fixa, 60,4\% (389/644) dos adolescentes do sexo masculino e $42,7 \%$ (208/487) do sexo feminino relataram fazer uso consistente do preservativo. Ao analisar o uso consistente entre aqueles que já haviam tido relações sexuais tanto com parceiro fixo, quanto com parceiro casual, as proporções observadas foram de 58,4\% (338/579) entre os meninos e de $43,5 \%(156 / 359)$ entre as meninas (dados não exibidos em tabela).

Quando questionados sobre os motivos que os levaram a ter a última relação sexual sem o uso de preservativo, muitos se esquivaram à res- posta. No entanto, considerando o total de adolescentes que já haviam iniciado a vida sexual ( $\mathrm{n}=2.106$ ), $24,5 \%$ disseram não ter usado preservativo na última relação sexual por confiarem no parceiro; $20,6 \%$, por falta de informação e/ou acesso; $16,7 \%$ relataram não gostar de usar o preservativo e $4,3 \%$ disseram que não o utilizaram porque seus amigos também não o utilizavam e/ou devido a outros motivos que não especificaram. Ainda com referência à última relação sexual, $4,1 \%$ dos adolescentes disseram ter tido relações sexuais porque estavam sob o efeito de bebidas alcoólicas.

Aplicando o modelo CHAID para analisar a consistência do uso de preservativos entre os indivíduos que referiram ter tido relação sexual com parceiro casual, obteve-se uma árvore composta por dois níveis e quatro nós (Figura 1).

A partir da inspeção visual da árvore assim gerada, podemos perceber que a variável que mais influencia o uso consistente/inconsistente de preservativo é o sexo do entrevistado. Olhando apenas para este nível, verificamos que as meninas apresentaram menor proporção de uso consistente de preservativos nas relações com parceiros casuais do que os meninos $(50 \% v s$. $63,7 \%$; 181/362 e 490/769, respectivamente). 
Freqüência do uso de drogas por gênero, segundo faixa etária de adolescentes de 12 municípios de Minas Gerais, Brasil, 2000.

\begin{tabular}{|c|c|c|c|c|}
\hline \multirow[t]{2}{*}{ Freqüência do uso } & \multicolumn{2}{|c|}{ Sexo } & \multirow[t]{2}{*}{ Total } & \multirow[t]{2}{*}{ Valor de $p$ * } \\
\hline & Masculino (\%) & Feminino (\%) & & \\
\hline \multicolumn{5}{|l|}{ Álcool } \\
\hline \multicolumn{5}{|l|}{$10-14$ anos $(n=2.691)$} \\
\hline Nunca usou & 54,1 & 54,1 & 54,1 & 0,915 \\
\hline Só experimentou/Não usa mais & 28,5 & 29,0 & 28,8 & \\
\hline Usa às vezes/Usa sempre & 17,4 & 16,9 & 17,1 & \\
\hline \multicolumn{5}{|l|}{$15-19$ anos $(n=2.949)$} \\
\hline Nunca usou & 27,9 & 34,4 & 31,9 & 0,000 \\
\hline Só experimentou/Não usa mais & 27,6 & 31,4 & 29,9 & \\
\hline Usa às vezes/Usa sempre & 44,5 & 34,2 & 38,2 & \\
\hline \multicolumn{5}{|l|}{ Cigarro } \\
\hline \multicolumn{5}{|l|}{$10-14$ anos $(n=2.595)$} \\
\hline Nunca usou & 76,9 & 77,1 & 77,0 & 0,872 \\
\hline Só experimentou/Não usa mais & 19,3 & 18,7 & 18,9 & \\
\hline Usa às vezes/Usa sempre & 3,9 & 4,2 & 4,1 & \\
\hline \multicolumn{5}{|l|}{$15-19$ anos $(n=2.820)$} \\
\hline Nunca usou & 61,4 & 63,7 & 62,8 & 0,296 \\
\hline Só experimentou/Não usa mais & 28,2 & 27,5 & 27,8 & \\
\hline Usa às vezes/Usa sempre & 10,3 & 8,8 & 9,4 & \\
\hline \multicolumn{5}{|l|}{ Maconha } \\
\hline \multicolumn{5}{|l|}{$10-14$ anos $(n=2.506)$} \\
\hline Nunca usou & 96,5 & 98,3 & 97,6 & 0,005 \\
\hline Usou pelo menos uma vez & 3,5 & 1,7 & 2,4 & \\
\hline \multicolumn{5}{|l|}{$15-19$ anos $(n=2.721)$} \\
\hline Nunca usou & 88,1 & 95,3 & 92,5 & 0,000 \\
\hline Usou pelo menos uma vez & 11,9 & 4,7 & 7,5 & \\
\hline \multicolumn{5}{|l|}{ Outra droga } \\
\hline \multicolumn{5}{|l|}{$10-14$ anos $(n=1.129)$} \\
\hline Nunca usou & 95,9 & 96,6 & 96,3 & 0,659 \\
\hline Usou pelo menos uma vez & 4,1 & 3,4 & 3,7 & \\
\hline \multicolumn{5}{|l|}{$15-19$ anos $(n=1.030)$} \\
\hline Nunca usou & 91,0 & 94,8 & 93,1 & 0,060 \\
\hline Usou pelo menos uma vez & 9,0 & 5,2 & 6,9 & \\
\hline
\end{tabular}

* Teste $\chi^{2}$ de independência.

O uso de drogas ilícitas parece modular o uso do preservativo entre os rapazes inseridos em relacionamentos casuais. Dos que referiram utilizar/ter utilizado alguma dessas substâncias, $55,7 \%(73 / 131)$ disseram usar preservativos de forma consistente, ao passo que, para os que nunca fizeram uso de drogas ilícitas, esse percentual foi de $65,4 \%$ (417/638).

Analisando comparativamente os três segmentos assim constituídos, temos que o segmento de rapazes que nunca utilizaram drogas ilícitas apresenta a maior proporção de uso consistente de preservativos, correspondendo a $65,4 \%$ (417/638). Já as meninas foram as que apresen- taram a maior proporção de uso não consistente de preservativos (Tabela 3 ).

O segundo modelo CHAID (Figura 2), referente ao uso de preservativos em relações com parceiros fixos, deu origem a uma árvore com três níveis e oito nós. Novamente, a característica que se mostrou mais relevante quanto ao uso do preservativo foi o sexo do entrevistado. Também, com referência às relações sexuais com parceiros fixos, os rapazes apresentaram maiores proporções de uso consistente do que as meninas, com uma proporção de 60,9\% (328/539), comparada a $42,3 \%$ (187/442) entre os meninos. 
Modelo chi-squared automatic interaction detector (CHAID) para uso de preservativo com parceiro casual.

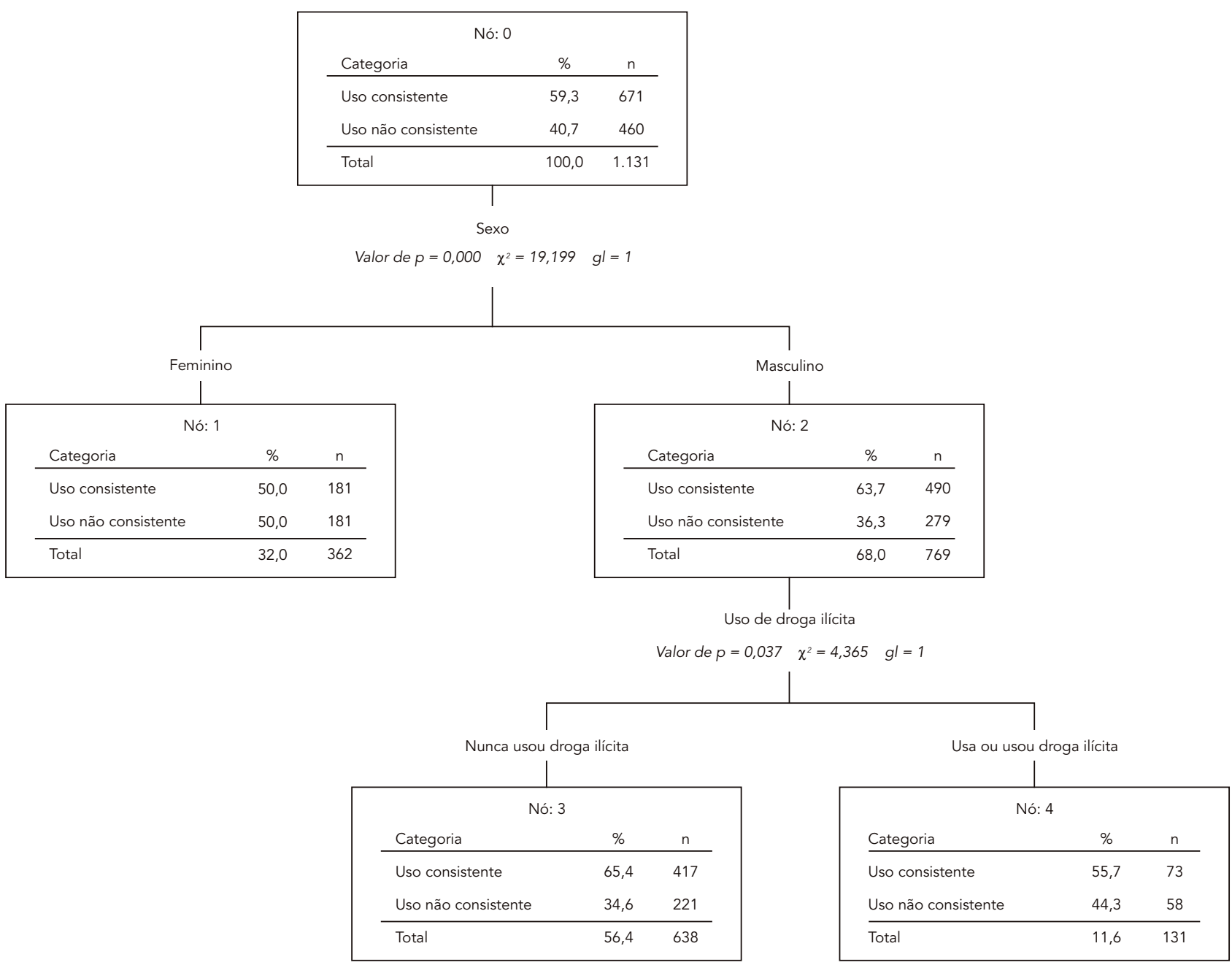

Com relação às mulheres, outro fator que influenciou a consistência do uso de preservativo em relações com parceiro fixo foi a faixa etária. Adolescentes da faixa de 15-19 anos parecem usar preservativos de forma menos consistente do que as de 10-14 anos. Enquanto 56,1\% (37/66) das mais novas referiram usar preservativo de forma consistente, essa proporção é de apenas $39,9 \%(150 / 376)$ entre as mais velhas.

Entre os rapazes, o uso de drogas se mostrou um fator determinante do uso do preservativo. Para os que já haviam feito ou faziam uso de alguma droga ilícita, o uso consistente foi referido por apenas $42,7 \%$ (35/82), enquanto para os que nunca fizeram uso dessas substâncias o percen- tual de uso consistente foi de $64,1 \%$ (293/457).

No nível subseqüente de particionamento dos dados, a variável referente ao uso de álcool e/ou cigarro foi discriminante apenas quanto ao subgrupo dos rapazes que nunca haviam utilizado drogas ilícitas. Neste nível de particionamento, o uso consistente do preservativo foi menos freqüente entre os que já haviam utilizado ou usam cigarro e/ou álcool, com uma proporção de 60,7\% (185/305), comparada a uma proporção de $71,1 \%(108 / 152)$ entre aqueles que referiram não utilizar álcool e cigarros.

Como exposto na Tabela 4, ao comparar o uso consistente de preservativos entre os diferentes segmentos, observamos que os adolescentes do 
Tabela 3

Segmentos populacionais segundo uso de preservativo com parceiro casual.

\begin{tabular}{lccc}
\hline $\begin{array}{l}\text { Segmentos populacionais } \\
(\mathbf{N}=1.131)\end{array}$ & $\begin{array}{c}\text { Uso consistente no } \\
\text { segmento }(\%)\end{array}$ & $\begin{array}{c}\text { Uso não consistente no } \\
\text { segmento (\%) }\end{array}$ & $\begin{array}{c}\text { População no } \\
\text { segmento (\%) }\end{array}$ \\
\hline Homens que nunca usaram droga ilícita $(n=638)$ & 65,4 & 34,6 & 56,4 \\
Homens que usam ou usaram droga ilícita $(n=131)$ & 55,7 & 44,3 & 11,6 \\
Mulheres ( $n=362)$ & 50,0 & 50,0 & 32,0 \\
\hline
\end{tabular}

Figura 2

Modelo chi-squared automatic interaction detector (CHAID) para uso de preservativo com parceiro fixo.

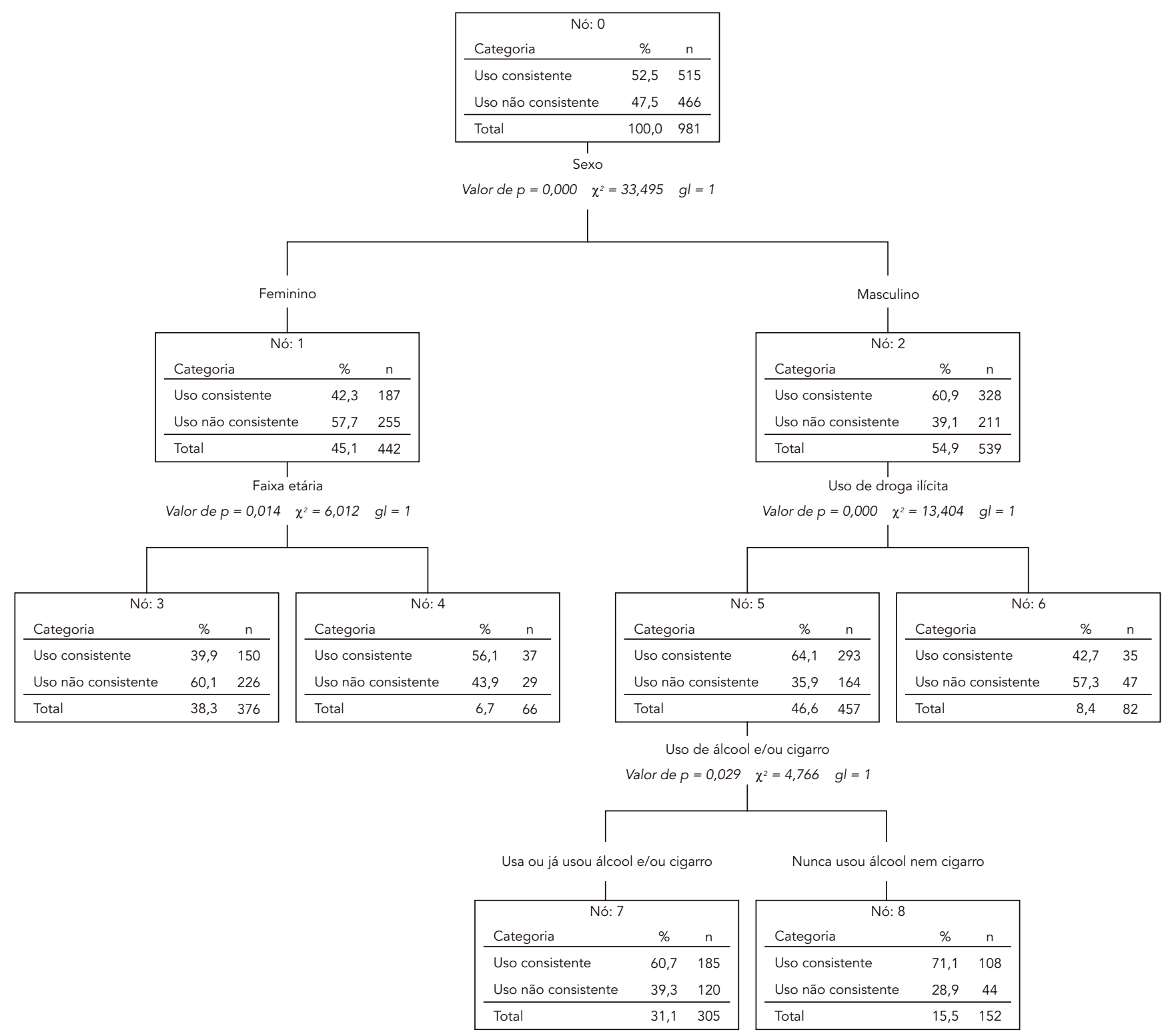


Segmentos populacionais segundo uso de preservativo com parceiro casual.

\begin{tabular}{|c|c|c|c|}
\hline $\begin{array}{l}\text { Segmentos populacionais } \\
(\mathrm{N}=981)\end{array}$ & $\begin{array}{l}\text { Uso consistente no } \\
\text { segmento (\%) }\end{array}$ & $\begin{array}{c}\text { Uso não consistente no } \\
\text { segmento (\%) }\end{array}$ & $\begin{array}{l}\text { População no } \\
\text { segmento (\%) }\end{array}$ \\
\hline \multicolumn{4}{|l|}{ Mulheres } \\
\hline $15-19$ anos $(n=376)$ & 39,9 & 60,1 & 38,3 \\
\hline $10-14$ anos $(n=66)$ & 56,1 & 43,9 & 6,7 \\
\hline \multicolumn{4}{|l|}{ Homens } \\
\hline \multicolumn{4}{|l|}{ Nunca usaram droga ilícita, nem álcool } \\
\hline e/ou cigarro $(n=152)$ & 71,1 & 28,9 & 15,5 \\
\hline \multicolumn{4}{|l|}{ Nunca usaram droga ilícita, porém usam ou } \\
\hline já usaram álcool e/ou cigarro $(n=305)$ & 60,7 & 39,3 & 31,1 \\
\hline Usam ou já usaram droga ilícita $(n=82)$ & 42,7 & 57,3 & 8,4 \\
\hline
\end{tabular}

sexo masculino que nunca haviam feito uso de substâncias lícitas ou ilícitas se mostraram como aqueles que apresentaram maiores proporções de uso consistente de preservativos $(71,1 \%$, 108/152). De modo oposto, o segmento das adolescentes com idades entre 15-19 anos foi o que apresentou a menor proporção de uso consistente de preservativos $(39,9 \%, 150 / 376)$.

Descrevendo os resultados de forma sucinta, tem-se que, tanto para os adolescentes com parceiros fixos, como para aqueles com parceiros casuais, a proporção de uso consistente de preservativos foi invariavelmente mais elevada entre jovens do sexo masculino (se comparados às do sexo feminino). Já entre os rapazes, essa proporção foi invariavelmente maior entre aqueles que nunca haviam feito uso de drogas.

\section{Discussão}

Os achados demonstram que o uso de substâncias psicoativas entre adolescentes da população estudada é bastante freqüente e que, mesmo no grupo dos mais jovens, esse consumo é relativamente prevalente. Estudos realizados em diferentes contextos corroboram os resultados aqui apresentados, alertando para o consumo freqüente de álcool, tabaco e outras drogas entre adolescentes $16,17,18,19$, porém tais comparações devem ser estabelecidas com cautela, em virtude de características particulares de cada amostra/ região estudada.

Carlini et al. 20, em pesquisa domiciliar envolvendo as 108 maiores cidades do país, descrevem que, entre adolescentes de 12-17 anos, $55 \%$ e $18,4 \%$ deles já haviam feito uso de álcool e tabaco, respectivamente. O uso de maconha entre indivíduos desta faixa etária foi de 3,6\%, exclusivamente entre adolescentes do sexo masculino. Os autores ressaltam ainda o precoce envolvimento dos adolescentes de 12-17 anos com as drogas, havendo, inclusive, relatos de situações de dependência e tratamento por consumo de tais substâncias.

De acordo com os resultados aqui apresentados, os padrões de uso de drogas não diferem de forma significativa segundo o sexo do adolescente: meninos e meninas apresentam padrões de consumo de álcool (exceto para a faixa etária de 15-19 anos), cigarro e drogas ilícitas (que não a maconha) relativamente similares. Feitas as ressalvas da não comparabilidade estrita dos diferentes estudos, resultados similares foram referidos por Galduróz et al. 21, em pesquisa com amostra representativa de indivíduos entre 12 65 anos, moradores de cidades com mais de 200 mil habitantes, em que, entre os adolescentes de 12-17 anos entrevistados, não houve diferença significativa entre indivíduos de ambos os sexos quanto ao consumo de álcool e tabaco.

Estudos anteriores realizados nos Estados Unidos e Brasil apontam para a associação entre o consumo de substâncias psicoativas e o uso não consistente de preservativos para a população em geral 22,23. Segundo os achados do presente trabalho, o mesmo parece ocorrer entre adolescentes, particularmente para os jovens do sexo masculino; estes mostraram uma maior proporção de uso não consistente de preservativos entre os que já haviam tido contato com algum tipo de droga, fosse ela lícita ou ilícita, tanto em relações com parceiros casuais como com parceiros fixos. Ou seja, parece haver, nesse caso, um efeito que perpassa os diferentes estratos de adolescentes do sexo masculino, embora não seja possível pre- 
cisar a direcionalidade da associação observada, se é que esta direcionalidade de fato existe. Coloca-se, portanto, em questão se adolescentes que fazem uso de substâncias psicoativas engajam-se com maior freqüência em práticas de risco, ou se esses jovens justificam e racionalizam a posteriori seus comportamentos de risco por causa do uso de psicoativos ("não sei direito, estava sob o efeito de drogas"). Ou ainda, se ambos os comportamentos são basicamente concomitantes, retroalimentando-se, conformando estilos de vida de maior risco e modos de interação social e afetiva de díades de parceiros 24,25.

Entre as moças, o uso consistente do preservativo é menos freqüente do que entre os rapazes, corroborando achados de outros estudos desenvolvidos entre adolescentes brasileiros 13,26,27,28. Porém, conforme a natureza desses relacionamentos, ou seja, a depender do fato de serem casuais ou fixos, observam-se diferenças substanciais quanto a essas proporções: com parceiros casuais, o uso consistente de preservativos foi de $50 \%$, ao passo que, com parceiros fixos, essa proporção se reduziu para 42,3\%. Segundo a literatura pertinente ao tema, isso se deveria a que, nos relacionamentos estáveis, a prioridade deixa de ser a proteção das infecções de transmissão sexual e passa ser a prevenção da gravidez, fazendo com que os preservativos sejam substituídos por outros métodos de contracepção, como a pílula anticoncepcional ${ }^{13}$. Em alguns casos, a proximidade afetiva faz com que, igualmente, a prevenção da gravidez seja relegada a segundo plano, tendo como conseqüência o aumento, anteriormente mencionado, de gravidezes nessa faixa etária.

O que perpassa todas essas situações são as relações de gênero nas quais meninas e meninos se acham inseridos, que contribuem para a maior vulnerabilidade das moças no âmbito das decisões relativas à vida sexual e reprodutiva 5 . Em geral, no contexto das regras culturais de nossa sociedade, ainda prevalece a perspectiva, mesmo que velada, de que as mulheres devem aceitar as condições impostas pelos homens para terem relações sexuais, com pouco espaço para negociar o uso de alguma forma de proteção contra o risco de contrair infecções de transmissão sexual e/ou gravidez indesejada 29.

É importante ressaltar que os resultados obtidos nesta pesquisa restringem-se a uma amostra de adolescentes regularmente matriculados em escolas públicas de Minas Gerais, que integraram a linha de base da avaliação de um programa de educação sexual implementado em alguns municípios daquele estado. Não podem, portanto, ser generalizados para a população de adolescentes como um todo, seja pelos diferentes contextos nas diversas localidades brasileiras, seja pelo fato de uma expressiva fração de adolescentes estar fora da escola. Em que pesem tais limitações, o estudo permite uma melhor compreensão acerca da influência do uso de álcool e drogas sobre as práticas sexuais entre adolescentes.

Os achados ressaltam a necessidade de implantar políticas públicas abrangentes, especialmente voltadas para adolescentes, a fim de que, cada vez mais, uma maior parcela dessa população passe a utilizar preservativos de forma consistente, com o intuito de prevenir não só a gravidez precoce, como também a transmissão do HIV/AIDS e demais IST. Todavia, exercendo o álcool e as drogas efeito modulador sobre o uso de preservativos, tal questão deve estar integrada a quaisquer ações preventivas no campo da saúde sexual e reprodutiva. De forma similar, as questões de gênero não podem ser jamais ignoradas na formulação dessas ações.

\section{Resumo}

Os achados provêm de um estudo transversal de 5.981 estudantes de escolas públicas de Minas Gerais, Brasil. Avaliou-se a influência do uso de drogas sobre as práticas sexuais. Dos rapazes com relacionamento casual que referiram ter utilizado drogas ilícitas, 55,7\% disseram usar preservativos de forma consistente (em todas as relações sexuais), enquanto entre os que nunca fizeram uso de tais substâncias, esse percentual foi de 65,4\%. Entre os rapazes com relacionamento fixo, que utilizaram droga ilícita, o uso consistente de preservativos foi referido por $42,7 \%$, ao passo que, para os que nunca fizeram uso dessas substâncias, esse percentual foi de 64,1\%. No subgrupo dos rapazes com par- ceria fixa que nunca utilizaram drogas ilícitas, o uso consistente do preservativo foi menos freqüente entre os que utilizaram cigarro elou álcool do que entre os que não referiram este uso (60,7\% vs. 71,1\%). As moças apresentaram menor proporção de uso consistente do preservativo do que os rapazes, independentemente do tipo de parceria, sem influência aparente dos padrões de consumo. Os achados sugerem a necessidade de integrar a prevenção do uso de drogas à de infecções sexualmente transmissiveis/gravidez indesejada.

Consumo de Bebidas Alcoólicas; Drogas Ilícitas; Comportamento Sexual; Adolescente 


\section{Colaboradores}

N. Bertoni foi responsável pela análise estatística e redação do texto. F. I. Bastos contribuiu com a elaboração e revisão do artigo. M. B. Mello participou da pesquisa empírica, elaboração e revisão do texto. M. Y. Makuch, M. H. Sousa, M. J. Osis e A. Faúndes contribuíram com a pesquisa empírica e revisão do manuscrito. Todos os autores discutiram, leram e aprovaram a versão final do artigo.

\section{Referências}

1. Tavares BF, Béria JU, Lima MS. Prevalência do uso de drogas e desempenho escolar entre adolescentes. Rev Saúde Pública 2001; 35:150-8.

2. Pratta EMM, Santos MA. Levantamento dos motivos e dos responsáveis pelo primeiro contato de adolescentes do ensino médio com substâncias psicoativas. Revista Eletrônica Saúde Mental Álcool e Drogas 2006; 2(2). http://www2.eerp.usp. $\mathrm{br} / \mathrm{resmad} / \mathrm{resmad} 4 /$ artigo_titulo.asp?rmr=81.

3. Galduróz JC, Noto AR, Nappo SA, Carlini EA. Trends in drug use among students in Brazil: analysis of four surveys in 1987, 1989, 1993 and 1997. Braz J Med Biol Res 2004; 37:523-31.

4. Tavares BF, Béria JU, Lima MS. Fatores associados ao uso de drogas entre adolescentes escolares. Rev Saúde Pública 2004; 38:787-96.

5. Villela WV, Doreto DT. Sobre a experiência sexual dos jovens. Cad Saúde Pública 2006; 22:2467-72.

6. Dias AB, Aquino EML. Maternidade e paternidade na adolescência: algumas constatações em três cidades do Brasil. Cad Saúde Pública 2006; 22:1447-58.

7. Brandão E, Heilborn ML. Sexualidade e gravidez na adolescência entre jovens de camadas médias do Rio de Janeiro, Brasil. Cad Saúde Pública 2006; 22:1421-30

8. Baraldi ACP, Daud ZP, Almeida AM, Gomes FA, Nakano AMS. Adolescent pregnancy: a comparative study between mothers who use public and private health systems. Rev Latinoam Enferm 2007; 15(spe):799-805.

9. Chalem E, Mitsuhiro SS, Ferri CP, Barros MC Guinsburg R, Laranjeira R. Gravidez na adolescência: perfil sócio-demográfico e comportamental de uma população da periferia de São Paulo, Brasil. Cad Saúde Pública 2007; 23:177-86.

10. Taquette SR, Vilhena MM, Paula MC. Doenças sexualmente transmissíveis e gênero: um estudo transversal com adolescentes no Rio de Janeiro. Cad Saúde Pública 2004; 20:282-90.

\section{Agradecimentos}

Estudo financiado pela Fundação Odebrecht.
11. Oliveira FA, Pfleger V, Lang K, Heukelbach J, Miralles I, Fraga F, et al. Sexually transmitted infections, bacterial vaginosis, and candidiasis in women of reproductive age in rural Northeast Brazil: a population-based study. Mem Inst Oswaldo Cruz 2007; 102:751-6.

12. Programa Nacional de DST e AIDS, Secretaria de Vigilância em Saúde, Ministério da Saúde. Pesquisa de conhecimento atitudes e práticas na população brasileira de 15 a 54 anos, 2004. Brasília: Ministério da Saúde; 2006

13. Teixeira AMFB, Knauth DR, Fachel JMG, Leal AF Adolescentes e uso de preservativos: as escolhas dos jovens de três capitais brasileiras na iniciação e na última relação sexual. Cad Saúde Pública 2006; 22:1385-96.

14. Viana FJM, Faúndes A, Mello MB, Sousa MH. Factors associated with safe sex among public school students in Minas Gerais, Brazil. Cad Saúde Pública 2007; 23:43-51.

15. Breiman L, Friedman J, Stone CJ, Olshen R. Classification and regression trees. Pacific Grove: Wadsworth; 1984.

16. Baus J, Kupek E, Pires M. Prevalência e fatores de risco relacionados ao uso de drogas entre escolares. Rev Saúde Pública 2002; 36:40-6.

17. Guimarães JL, Godinho PH, Cruz R, Kappann JI, Tosta Júnior LA. Consumo de drogas psicoativas por adolescentes escolares de Assis, SP. Rev Saúde Pública 2004; 38:130-2.

18. De Micheli D, Formigoni ML. Drug use by Brazilian students: associations with family, psychosocial, health, demographic and behavioral characteristics. Addiction 2004; 99:570-8.

19. Costa MCO, Alves MVQM, Santos CAST, Carvalho RC, Souza KEP, Sousa HL. Experimentação e uso regular de bebidas alcoólicas, cigarros e outras substâncias psicoativas (SPA) na adolescência. Ciên Saúde Coletiva 2007; 12:1143-54. 
20. Carlini EA, Galduróz JCF, Noto AR, Nappo SA. II Levantamento domiciliar de drogas psicotrópicas no Brasil: estudo envolvendo as 108 maiores cidades do país - 2005. São Paulo: Centro Brasileiro de Informações sobre Drogas Psicotrópicas/Universidade Federal Paulista; 2005.

21. Galduróz JCF, Noto AR, Nappo SA, Carlini EA. Uso de drogas psicotrópicas no Brasil: pesquisa domiciliar envolvendo as 107 maiores cidades do país - 2001. Rev Latinoam Enferm 2005; 13(spe): 888-95.

22. Demographic and behavioral predictors of sexual risk in a multisite HIV prevention trial. NIMH Multisite HIV Prevention Trial. AIDS 1997; 11 Suppl 2: S21-7.

23. Bastos FI, Cunha CB, Bertoni N. Uso de substâncias psicoativas e métodos anticoncepcionais pela população urbana brasileira, 2005. Rev Saúde Pública 2008; 42 Suppl 1:118-26.

24. Fortenberry JD. Alcohol, drugs, and STD/HIV risk among adolescents. AIDS Patient Care STDS 1998; 12:783-6.
25. Woodrome SE, Zimet GD, Orr DP, Fortenberry JD. Dyadic alcohol use and relationship quality as predictors of condom non-use among adolescent females. J Adolesc Health 2006; 38:305-6.

26. Rocha CLA, Horta BL, Pinheiro RT, Cruzeiro ALS, Cruz S. Use of contraceptive methods by sexually active teenagers in Pelotas, Rio Grande do Sul State, Brazil. Cad Saúde Pública 2007; 23:2862-8.

27. Martins LBM, Costa-Paiva LHS, Osis MJD, Sousa MH, Pinto-Neto AM, Tadini V. Fatores associados ao uso de preservativo masculino e ao conhecimento sobre DST/AIDS em adolescentes de escolas públicas e privadas do Município de São Paulo, Brasil. Cad Saúde Pública 2006; 22:315-23.

28. Szwarcwald CL, Barbosa-Júnior A, Pascom AR, Souza-Júnior PR. Knowledge, practices and behaviours related to HIV transmission among the Brazilian population in the 15-54 years age group, 2004. AIDS 2005; 19 Suppl 4:S51-8.

29. Heise L, Ellsberg M, Gottmoeller M. A global overview of gender-based violence. Int J Gynaecol Obstet 2002; 78 Suppl 1:S5-14.

Recebido em 25/Mar/2008

Versão final reapresentada em 29/Out/2008 Aprovado em 21/Jan/2009 